\title{
Food imprinting in the domestic chick:
}

\section{A reconsideration}

MERIE: $E$. MEYER and LAWRENCE $H$. FRANK, Western Washington State College, Bellingham, Wash. 98225

Liverimental chickswere trained to peck "gre'n stimulas for fored in preference to a blue stimulus at the ages of 1,3 , or 5 days old within a socialized or isolated condition. The birds were tested on four subsequent days without food in order to ascertain the generality of' "food imprinting." On the initial reinforced day, the chicks discriminated between the two stimuli, older birds pecked more than younger ones, and those within the socialized condition more than the isolated ones. A rapid decline in the frequency of responses over the nonreinforced days was interpreted as extinction of a discriminative response rather than as "food imprinting."

Recently, Hess (1964) has extended the principles of imprinting to that of food imprinting and has distinguished between imprinting and associative learning. He has argued that in imprinting certain classes of stimuli to which the chick has been exposed during the critical period continue to function as unconditioned stimuli without a decline in the response strength. Once the critical period has been passed without imprinting, these same stimuli do not function as unconditioned stimuli but rather as neutral ones. However, these stimuli can function as potential conditioned stimuli through associative learning. Furthermore, Hess has emphasized that imprinting and associative learning differ in that ".. . these two categories of responses are completely different in terms of their long-range effects on the character of the animal's behavior [p. 1138] ."

In a series of experiments, Tolman $(1964,1965)$ has investigated the function of social facilitation upon feeding behavior of domestic chicks. He has observed that young chicks eat more in groups than in social isolation and that feeding was related to unrestricted interaction between chicks. Hess has maintained that social stimuli are irrelevant to food imprinting. However, in his experiments on food imprinting, the chicks were imprinted in groups. Hence the

Fig. 1. Per cent of responses of control chicks for pecking the green key at 1,3 , or 5 days of age in an isolated or socialized group. distance between the apertures of the two stimuli was $4.5 \mathrm{~cm}$. Behind each aperture was a plastic holder that could contain food. When the chick pecked the stimulus, it activated a microswitch (RW8435, Microswitch Company) that, in turn, was recorded on an individual counter.

$$
\text { PROCEDURE AND }
$$

possibility arises that his data were in part present research constitutes a further examination of the function of social facilitation upon food imprinting.

\section{SUBJECTS}

The Ss were 192 white Leghorn cockerel chicks (Kimber) and were obtained from a local commercial hatchery and brought into the laboratory on the day of hatching. Within the laboratory, the chicks were maintained in groups of 25 in a $30^{\circ} \mathrm{C}$ brooder box. The food used in maintenance and during the experimental sessions was Albers Trip-L-Duty Paketts poultry feed. Food and water were available except for a 4-h food-deprivation period each day prior to being placed in the experimental box.

\section{APPARATUS}

The apparatus was a modified Hess pecking preference box. It was constructed in such a manner that Masonite panels could be inserted to convert the $40 \times 10 \mathrm{~cm}$ box into four $10 \times 10 \mathrm{~cm}$ individual compartments. The inside walls of the box were $28 \mathrm{~cm}$ high and were painted a flat neutral gray. The floor was covered with hardware cloth and the top of the box was open. On the common side of each of the individual compartments were two stimuli, a green circle and a blue circle $2.5 \mathrm{~cm}$ in diam, and in the center of each stimulus was a $6-\mathrm{mm}$ aperture. The aperture was $5.8 \mathrm{~cm}$ above the floor and

\section{EXPERIMENTAL DESIGN}

The experimental chicks were divided in to three age levels of 1,3 , or 5 days old, and within each age level the chicks were assigned to either the social or the isolated condition. Socialized Ss were those run in a group of four birds; isolated Ss were run in individual compartments. Sixteen chicks were in each of the six groups. At the appropriate age level, the birds were deprived of food 4-h before a 4-h experimental session. During the Day 1 session, pecking the aperture of the green circle was reinforced with food. On the following 4 days, the birds were deprived of food 4-h before the 4-h experimental session. However, no food was available during the experimental session. Responses to the blue stimuli were not reinforced at any time throughout the experimental sessions. The number of responses to both the green and blue stimuli were recorded separately.

The procedure and experimental design for the control chicks were identical to that for the experimental groups with one exception. During the Day 1 session, pecking the aperture of the green circle was not reinforced with food as it was for the experimental groups.

RESULTS AND DISCUSSION

Following Hess's analysis, the per cent of response to the green stimuli for the

\section{CONTROL GROUP}

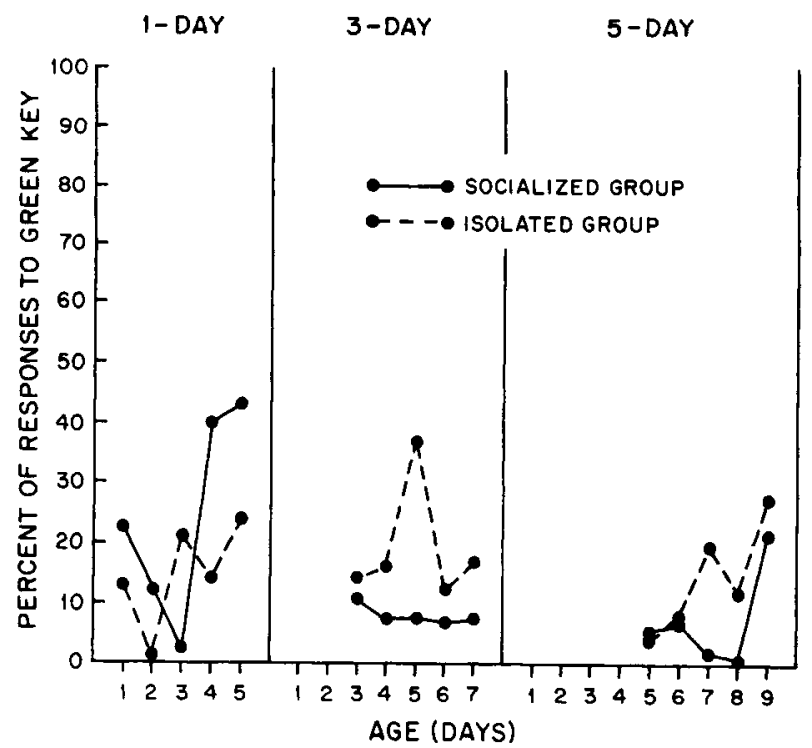


Fig. 2. Mean number of responses of control chicks for pecking the green key at 1 . 3 , or 5 days of age in an isolated or socialized group.

various control groups is shown in Fig. 1. As can be observed from the figure, all control birds showed a response preference for the blue stimulus. These data are confirmatory and yield substantially the same conclusion as reported by Hess.

The mean number of responses for the control groups to the green and blue stimuli within the socialized and isolated groups over days is given in Fig. 2. From this figure, it can be seen that while the preference was for the blue stimuli, the mean responses over groups were low. In comparison to the isolated groups, the socialized birds responded more on the initial day. However, on the following days, the mean response rate declined. The per cent of responses to the green or reinforced stimuli for the experimental groups is shown in Fig. 3. Within the socialized groups, the per cent of responses following the initial reinforced day declined for the 1- and 5-day-old groups. However, like Hess's birds, the 3-day-old group maintained a high per cent of responses to the green stimulus throughout the experimental sessions. For the most part, the results for the socialized groups of the present experiment replicated Hess's findings. The data for the 3-day-old group with an analysis using percentage values can be interpreted as evidence for food imprinting. On the other hand, the 1 -day-old birds that were run as isolates responded more to the blue stimulus over days, while the 3-and 5-day-old isolated groups showed a strong preference in per cent of responses to the green stimulus during the reinforced day. On subsequent nonreinforced days, the 3- and 5-day-old isolated groups showed a decrease in per cent of responses to the previously reinforced stimulus. It is readily apparent that chicks can be imprinted to food, but that food imprinting is a function of social facilitation. When the isolated chick is exposed to food during the 3rd day of age and food is withdrawn on the next 4 days during the test sessions, extinction occurs rather than imprinting.

The mean frequencies of the pecking responses for the experimental groups to the green and blue stimuli over days within the socialized or isolated group conditions are shown in Fig. 4. As it can be observed

Fig. 3. Per cent of responses of experimental chicks reinforced for pecking the green key at 1,3 , or 5 days of age in an isolated or socialized group.

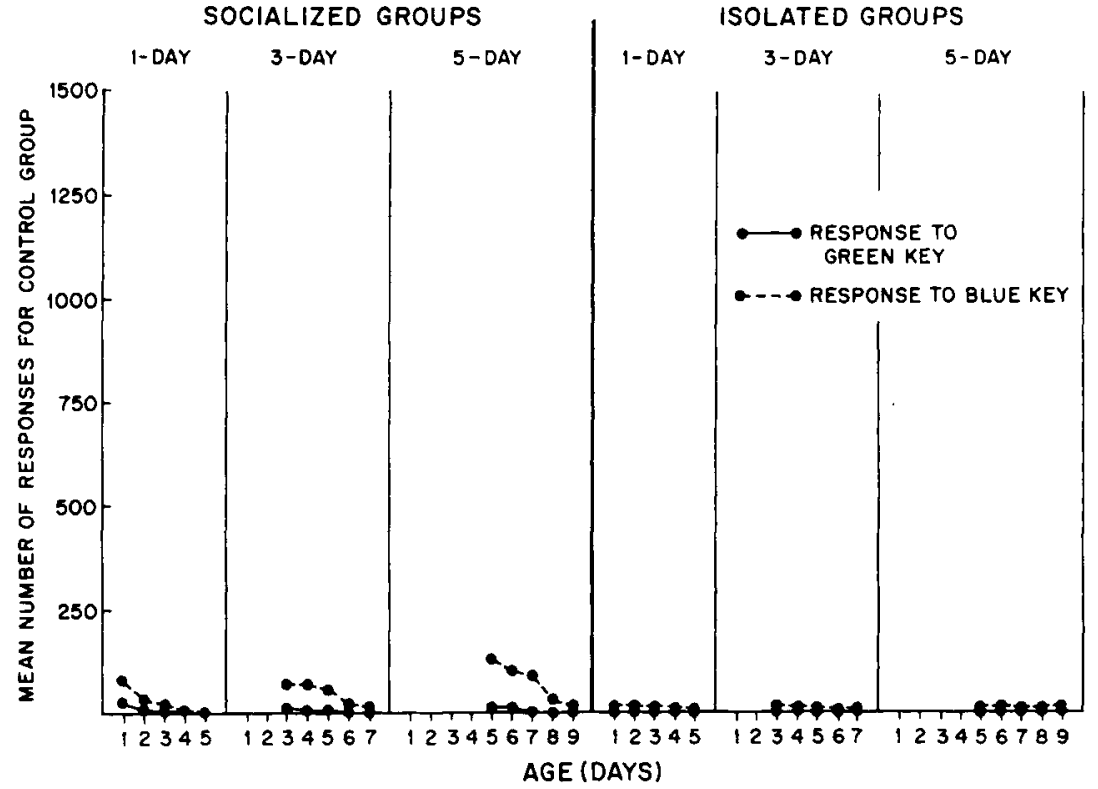

from this figure, chicks that were older pecked more on the initial or reinforced day in both the socialized and isolated groups than did the younger birds. However, there are marked differences between the two treatment groups. Chicks in the socialized groups responded at a much higher rate than did the isolated chicks. It appears from these data that the rate of responses was greatly facilitated for all ages by the socialized condition. Furthermore, it may be observed that in both the isolated and socialized experimental chicks, they showed a strong preference for the reinforced green stimulus over the preferred blue stimulus of the control groups. Only within the

isolated 1-day-old experimental group did the preference for the green reinforced stimulus break down and the chicks tended to respond more to the blue stimulus.

One of the most salient features in Fig. 4 is the rapid decline in the frequency of response over the nonreinforced days. This decrease in responses occurred to both the green and blue stimuli, but was most pronounced with the responses associated with the green or the prior reinforcing stimulus. These data may be interpreted as extinction of a discriminative stimulus and are inconsistent with an imprinting interpretation.

The general results of the present study are similar to that expected on the basis of

\section{EXPERIMENTAL GROUP}

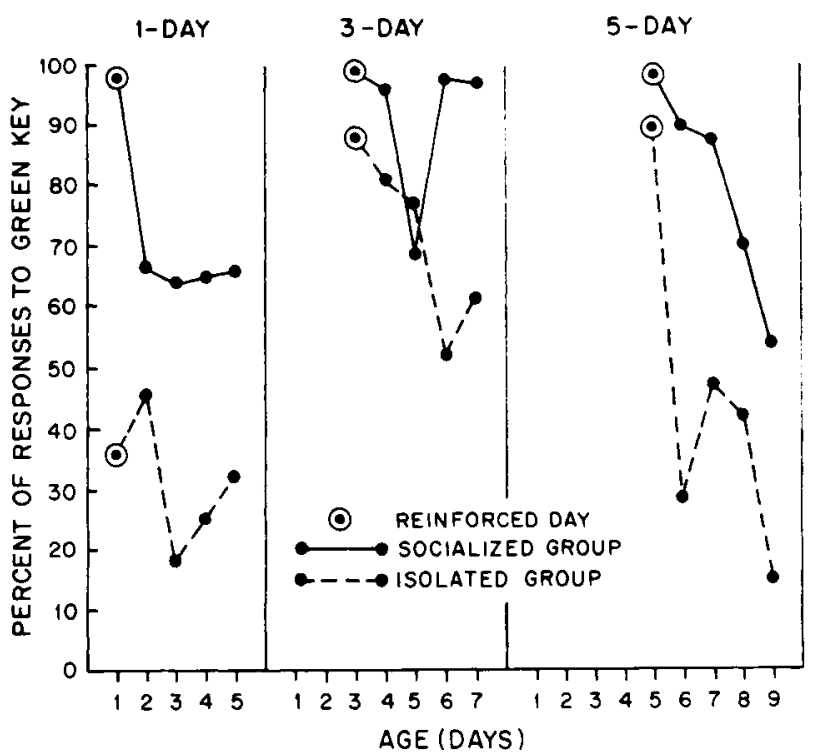




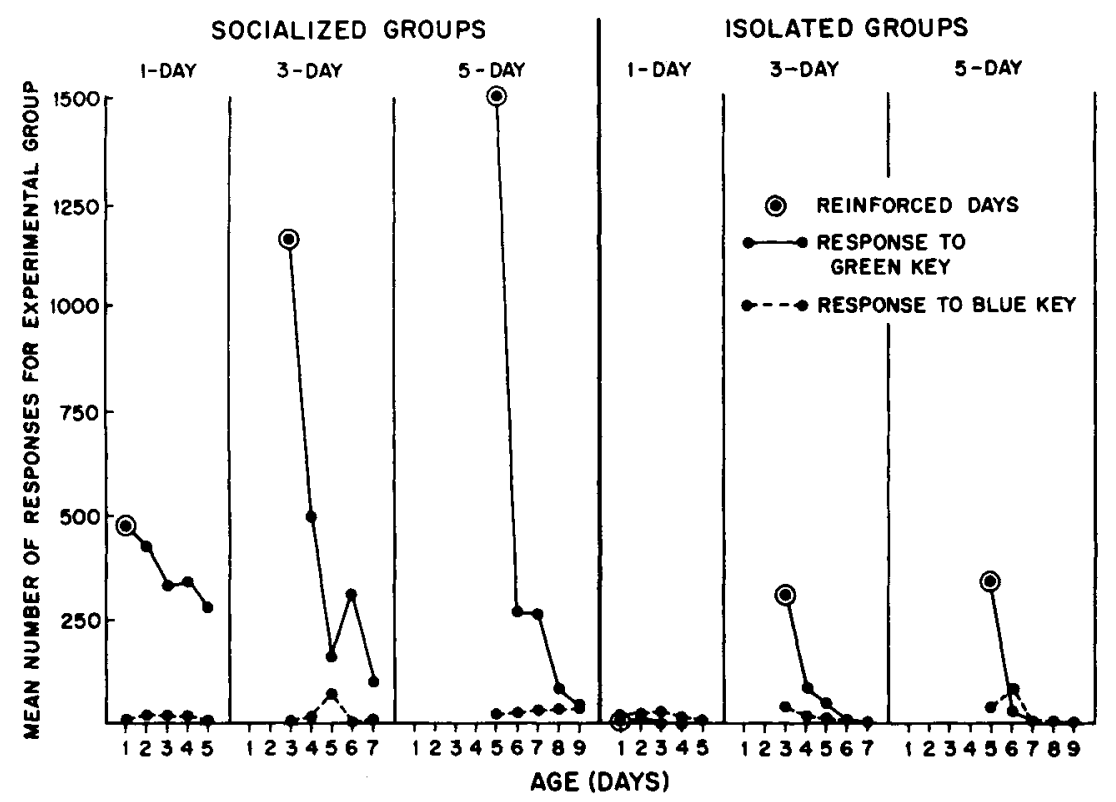

\section{Discriminative effect of fixed-ratio feeder flash on DRL responding ${ }^{1}$}

JAY S. MILLER, T. A. MCCULLOUGH, and DONALD M. THOMPSON, ${ }^{2}$ University of Maryland, College Park, Md. 20742

Two pigeons were initially trained under a DRL 20-sec schedule of grain reinforcement. Fixed-ratio 15 or 20) schedules of 0.5-sec magazine presentation (feeder flash) were then superimposed upon the DRL schedule. During the initial rate increase and the subsequent return to baseline, a significantly large proportion of the DRL reinforcements was obtained by the first response following a feeder flash.

Zimmerman (1963) demonstrated that when pigeons' responses on one key were reinforced with food, responding on a second key could be maintained indefinitely solely by presentations of the feeder magazine and associated stimuli for periods of time so brief $(0.5 \mathrm{sec})$ as to preclude the obtaining of any food (feeder flash). In a single key situation, Randolph \& Sewell (1965) superimposed a fixed-ratio (FR) 10 schedule of feeder-flash presentation upon responding maintained by a differential-reinforcement-of-low-rate (DRL) 20-sec schedule of grain reinforcement. Such a situation, in which two schedules operate simultaneously and independently on the same operandum, has been termed a conjoint schedule (Catania, et al, 1966). Randolph \& Sewell (1965) and Stubbs (1967), in a subsequent replication, found that response rate increased during probe sessions in which the conjoint schedule was in effect. This increased DRL rate was correlated with a decrease in the number of food reinforcements obtained. Pigeons with efficient, low DRL baseline rates showed lower rate increases during the probe sessions.

The present experiment was begun as part of a larger series of experiments examining the Randolph \& Sewell (1965) procedure. During initial probe sessions, however, it was fortuitously noted that the pigeons were obtaining a large proportion of DRL reinforcements for the first response following the feeder flash. This relationship is not apparent from the data presented by Randoiph \& Sewell (1965) and Stubbs (1967). Our observation suggested that the feeder flash had discriminative properties for DRL responding, and a systematic examination of the effect was made.

\section{SUBJECTS}

The Ss were two male, experimentally naive White Carneaux pigeons. The pigeons were maintained at $80 \%$ of free feeding weight. Water was always available in the home cages.
APPARATUS
A standard pigeon chamber (Ferster \&

Fig. 4. Mean number of responses of experimental chicks reinforced for pecking the green key at 1,3 , or 5 days of age in an isolated or socialized group.

maturation, social facilitation, and associative learning. When the data are in total frequencies, the interpretation of good imprinting is not warranted and may be better explained in terms of associative learning. Food imprinting occurred only for the 3-day-old socialized groups when the data are transformed into percentage values.

\section{REFERENCES}

HESS, E. H. Imprinting in birds. Science, 1964, 146, 1128-1139.

TOLMAN, C. W. Social faciliation of feeding behavior in the domestic chick. Animal Behaviour, 1964, 12, 245-251.

TOLMAN, C. W., \& WILSON, G. F. Social feeding in domestic chicks. Animal Behaviour, $1965,13,134-142$.

Skinner, 1957) was located in a sound attenuating box. A Gerbrands response key was mounted in the center of the front panel, $25 \mathrm{~cm}$ above the wire floor, and was lit by a yellow 6-W bulb. The aperture of the grain magazine was directly below the key. A small metal "lip" at the bottom of the aperture prevented any grain from spilling out. Presentation of the magazine was accompanied by the offset of the key light, the noise of solenoid operation, and the onset of a white light located inside the magazine. This light remained on throughout the feeder cycle. Feeder cycles of two different durations were used: a 4-sec (regular) cycle and a 0.5 -sec (feeder flash) cycle. White noise was continually present in the experimental room. Data were recorded on a cumulative recorder, digital counters, and a Sodeco printing counter.

\section{PROCEDURE}

All sessions were $1 \mathrm{~h}$ long and on consecutive days. On the first day, the birds were magazine trained, shaped to key peck, and given 25 reinforcements for pecking. On the following day, responses were reinforced under a DRL 20-sec schedule of reinforcement. Under this schedule, a response is reinforced if $20 \mathrm{sec}$ or more have elapsed since the last response or since the termination of the magazine cycle if the previous response was reinforced. This schedule remained in effect until responding was judged to be stable across a 5-day period. Pigeon 9 then received 13 sessions in which an FR 10 schedule of feeder-flash reinforcement was superimposed upon the DRL 20-sec schedule for regular reinforcement (Conjoint DRL 20 FR 10). When a single 